\title{
Is Polymicrobial Bacteremia an Independent Risk Factor for Mortality in Acinetobacter baumannii Bacteremia?
}

\author{
Yung-Chih Wang ${ }^{1}{ }^{\mathbb{D}}$, Wen-Wei Ku ${ }^{2}$, Ya-Sung Yang ${ }^{1}$, Chih-Chun Kao ${ }^{3}$, Fang-Yu Kang ${ }^{3}$, \\ Shu-Chen Kuo ${ }^{4}$, Chun-Hsiang Chiu ${ }^{1}$, Te-Li Chen ${ }^{5,6}$, Fu-Der Wang ${ }^{6,7}$ and Yi-Tzu Lee ${ }^{3,7, *}$ (1) \\ 1 Division of Infectious Diseases and Tropical Medicine, Department of Internal Medicine, Tri-Service General \\ Hospital, National Defense Medical Center, Taipei 11490, Taiwan; wystwyst@gmail.com (Y.-C.W.); \\ ysyoung4097@gmail.com (Y.-S.Y.); pipi10279@gmail.com (C.-H.C.) \\ 2 Division of Infectious Diseases, Department of Medicine, Taipei City Hospital Renai Branch, Taipei 10629, \\ Taiwan; DAN77@tpech.gov.tw \\ 3 Department of Emergency Medicine, Taipei Veterans General Hospital, Taipei 11217, Taiwan; \\ rosty.kao@gmail.com (C.-C.K.); lynnkang8043@hotmail.com (F.-Y.K.) \\ 4 National Institute of Infectious Diseases and Vaccinology, National Health Research Institute, \\ Maoli County 35053, Taiwan; ludwigvantw@gmail.com \\ 5 Graduate Institute of Life Sciences, National Defense Medical Center, Taipei 11490, Taiwan; \\ tecklayyy@gmail.com \\ 6 Division of Infectious Diseases, Taipei Veterans General Hospital, Taipei 11217, Taiwan; \\ fdwang@vghtpe.gov.tw \\ 7 Faculty of Medicine, School of Medicine, National Yang-Ming University, Taipei 11221, Taiwan \\ * Correspondence: s851009@yahoo.com.tw; Tel.: +886-2-28757628; Fax: +886-2-28730052
}

Received: 2 December 2019; Accepted: 31 December 2019; Published: 6 January 2020

\begin{abstract}
This retrospective observational study assessed the differences between monomicrobial and polymicrobial A. baumannii bacteremia and identified possible independent risk factors for 14-day mortality. There were 379 patients with A. baumannii bacteremia admitted to a tertiary care center in northern Taiwan between August 2008 and July 2015 enrolled for data analysis. Among them, 89 patients $(23.5 \%)$ had polymicrobial bacteremia and 290 patients $(76.5 \%)$ had monomicrobial bacteremia. No significant difference in 14-day mortality was observed between patients with monomicrobial and polymicrobial $A$. baumannii bacteremia ( $26.9 \%$ vs. $29.2 \%, p=0.77$ ). Logistic regression controlled for confounders demonstrated that polymicrobial bacteremia was not an independent predictor of mortality, whereas appropriate antimicrobial therapy was independently associated with reduced mortality. Higher 14-day mortality rates were observed in the polymicrobial bacteremic patients with concomitant isolation of Escherichia coli, Pseudomonas aeruginosa, and Enterobacter spp. from the bloodstream. Compared with patients with monomicrobial multidrug-resistant $A$. baumannii (MDRAb) bacteremia, those with MDRAb concomitant with Gram-negative bacilli bacteremia had a worse outcome. Polymicrobial A. baumannii bacteremia was not associated with a higher 14-day mortality rate than that of monomicrobial A. baumannii bacteremia, although more deaths were observed when certain Gram-negative bacteria were concomitantly isolated. Appropriate antimicrobial therapy remains an important life-saving measure for A. baumannii bacteremic patients.
\end{abstract}

Keywords: Acinetobacter baumannii; appropriate therapy; bacteremia; mortality; polymicrobial infection 


\section{Introduction}

Polymicrobial bacteremia occurs in $6 \%-27.4 \%$ of patients with bloodstream infections and was first identified as an important problem in the 1960s for its higher attributable mortality rate compared with that for monomicrobial bacteremia [1-4]. The mortality rate of patients with polymicrobial bacteremia varied from $36 \%$ to $63 \%$ [1-4]. This diverse mortality rate may be associated with heterogeneity in the comorbid conditions of the patients [2,5,6], varied infection sources [7], and different causative pathogens $[2,3,5,6]$.

Acinetobacter baumannii is an emergent nosocomial pathogen with high morbidity and mortality rates [8-10]. Bacteremia caused by A. baumannii occurs as polymicrobial infections in $19 \%-35 \%$ of cases $[6,9,11,12]$. Our previous study found that carbapenem-resistant $A$. baumannii (CRAb) provided a sheltering effect for carbapenem-susceptible pathogens and enhanced the pathogenesis of polymicrobial infection [13]. However, no study has evaluated the impact of polymicrobial A. baumannii bacteremia on clinical outcomes. Therefore, this retrospective observational study assessed whether polymicrobial infection was an independent risk factor for 14-day mortality in patients with A. baumannii bacteremia.

\section{Materials and Methods}

\subsection{Study Population}

This retrospective study was conducted at Taipei Veterans General Hospital (T-VGH), a 2900-bed tertiary-care teaching hospital located in Taipei, Taiwan, during a seven-year period from August 2008 to July 2015. Charts were reviewed for all patients with at least one blood culture positive for Acinetobacter calcoaceticus-Acinetobacter baumannii (Acb) complex accompanied by two or more of the following symptoms and signs of infection: fever, hypothermia, tachypnea, tachycardia, leukocytosis, or leukopenia. We included only the first blood culture for patients with two or more positive blood cultures. Patients under 20 years of age and those with incomplete medical records were excluded. The protocol was approved by the institutional review boards (IRBs) of T-VGH (IRB No. 2016-05-019CC).

\subsection{Data Collection}

We reviewed the medical records to retrieve clinical information, including demographic characteristics, comorbidities, duration of intensive care unit (ICU) and hospital stays, time of receipt, dose and route of therapy with individual antimicrobial drugs, receipt of invasive procedures, time of bacteremia onset, and infectious foci. The onset of bacteremia was defined as the day when the blood culture that eventually yielded A. baumannii was drawn. Polymicrobial bacteremia was defined as the simultaneous isolation of $A$. baumannii and one or more microorganisms from the blood during the same bacteremic episode. However, Corynebacterium spp., Bacillus spp., Propionibacterium acnes, and skin flora (e.g., Micrococcus spp., Streptococcus viridans, and coagulase-negative staphylococci) were considered contaminants and not included in the analyses unless they were related to device infection and grown in two or more blood cultures [6,14]. Episodes of bloodstream infection were defined as ICU-acquired bacteremia if they appeared $48 \mathrm{~h}$ after ICU admission. Chronic kidney disease was defined as an estimated glomerular filtration rate $<60 \mathrm{~mL} / \mathrm{min} / 1.73 \mathrm{~m}^{2}$. Neutropenia was defined as an absolute neutrophil count $<0.5 \times 10^{9}$ neutrophils/L. Immunosuppressive therapy was defined as the receipt of cytotoxic agents within six weeks [15], corticosteroids at a dosage equivalent to or higher than $15 \mathrm{mg}$ of prednisolone daily for at least seven days within four weeks, or other immunosuppressive agents within two weeks before bacteremia onset $[15,16]$. A history of recent surgery was defined as receipt of operations within four weeks before bacteremia onset. The severity of infection was evaluated using the Acute Physiology and Chronic Health Evaluation (APACHE) II score [17] within $24 \mathrm{~h}$ before bacteremia onset. The primary infection source of bacteremia was determined according to the definitions from the Centers for Disease Control and Prevention [18]. Appropriate antimicrobial therapy was defined as the administration of at least one antimicrobial agent to which the causative pathogen was susceptible in vitro within $48 \mathrm{~h}$ after the onset of bacteremia, with an approved route and 
dosage appropriate for end organ(s) function. In patients with polymicrobial bacteremia, a combination of antimicrobial agents covering all isolated microbes was deemed appropriate. Monotherapy with an aminoglycoside was not considered appropriate therapy for A. baumannii bacteremia. The primary outcome measure was all-cause 14-day mortality following bacteremia onset. Fourteen-day mortality was chosen as the endpoint to allow adequate ascertainment of treatment response. We reasoned that 30 days is too long for critically ill patients, as there are many competing causes of death, and seven days is too short a time to witness a response to treatment.

\subsection{Microbiological Studies}

Bacteria were phenotypically identified to Acb complex using a Vitek 2 system (bioMérieux, Marcy l'Etoile, France). Other bacterial species were also identified using this system. All isolates were regrown from $-80{ }^{\circ} \mathrm{C}$ storage, identified to the species level, and tested for their susceptibility to various antimicrobials. A multiplex polymerase chain reaction (PCR) method was used to identify A. baumannii to the genomic species level [19]. Antimicrobial susceptibilities of A. baumannii and other concomitant isolated bacterial pathogens were determined by the agar dilution method [20] and interpreted according to the Clinical Laboratory Standards Institute (CLSI) criteria [21].

Multidrug resistance was defined as resistance to three or more of the following classes of antimicrobials: anti-pseudomonal cephalosporins, anti-pseudomonal carbapenems, ampicillin/sulbactam, fluoroquinolones, and aminoglycosides. Carbapenem resistance was defined as resistance to imipenem, meropenem, or doripenem.

\subsection{Statistical Analysis}

Chi-square tests with Yates correction or Fisher's exact tests were used to compare categorical variables; Student's t or Mann-Whitney rank-sum tests were used to analyze continuous variables, as appropriate. Logistic regression models were used to explore independent risk factors of 14-day mortality. We performed univariate analyses separately for each risk factor to ascertain the odds ratios (ORs) and 95\% confidence intervals (CIs). All biologically plausible variables with a $p<0.20$ in the univariate analysis were considered for inclusion in the logistic regression model in the multivariable analysis. A backward selection process was utilized. Interactions between APACHE II score and the covariates were also examined in the logistic regression model. Time to mortality, defined as the interval between bacteremia onset and death, was analyzed by Kaplan-Meier survival analysis with log-rank tests. $p<0.05$ was considered statistically significant. SPSS Statistics for Windows, version 22.0 (IBM Corp., Armonk, NY, USA) was used for all data analyses.

\section{Results}

During the seven-year study period, we enrolled 732 patients with at least one set of blood cultures positive for Acb complex. A total of 379 patients met the inclusion criteria, of which $89(23.5 \%)$ patients had polymicrobial $A$. baumannii bacteremia. The demographic and clinical characteristics of patients with polymicrobial and monomicrobial $A$. baumannii bacteremia are summarized in Table 1 . Patients with polymicrobial $A$. baumannii bacteremia were older, more likely to have coronary artery disease, less frequently experienced shock within three days of bacteremia onset, and more frequently received inappropriate antimicrobial therapy $(13.5 \%$ vs. $38.6 \%, p<0.001)$, compared with those in patients with monomicrobial bacteremia. No significant difference in 14 -day mortality $(29.2 \%$ vs. $26.9 \%, p=0.770)$ and 30 -day mortality $(38.2 \%$ vs. $34.8 \%, p=0.561)$ was observed between these two groups. To analyze the duration of in-hospital stay after the onset of bacteremia, we separated the population into two groups, survivors and non-survivors, at hospital discharge. In the patients who survived to discharge, those with polymicrobial A. baumanii bacteremia and monomicrobial A. baumanii bacteremia had a comparable hospital stay after the onset of bacteremia (21.9 [9-35] vs. 21.9 [9-34] days, $p=0.997)$. In terms of the patients who died in hospital, those with polymicrobial A. baumanii bacteremia and monomicrobial $A$. baumanii bacteremia also had a comparable hospital stay after the onset of bacteremia 
(13.9 [0.75-26.5] vs. 13.7 [1-21] days, $p=0.944)$. The 30-day re-admission rates of survived patients with polymicrobial and monomicrobial A. baumannii bacteremia were not significantly different $(17.9 \%$ and $13.2 \%$, respectively; $p=0.620$ ).

Table 1. Demographic and clinical characteristics of patients with monomicrobial and polymicrobial Acinetobacter baumannii bacteremia.

\begin{tabular}{|c|c|c|c|c|}
\hline Characteristic & $\begin{array}{c}\text { All } \\
(n=379)\end{array}$ & $\begin{array}{c}\text { Polymicrobial } \\
\quad(n=89)\end{array}$ & $\begin{array}{c}\text { Monomicrobial } \\
\quad(n=290)\end{array}$ & $p$ \\
\hline \multicolumn{5}{|l|}{ Demographic characteristics } \\
\hline Age, years & $74(60-81)$ & $78(64-82.5)$ & $73(59-81)$ & 0.012 \\
\hline Male sex & $280(73.9)$ & $66(74.2)$ & $214(73.8)$ & 1.000 \\
\hline Community-onset & $20(5.3)$ & $2(2.2)$ & $18(6.2)$ & 0.181 \\
\hline Acquired in ICU & $189(49.9)$ & $47(52.8)$ & $142(49.0)$ & 0.608 \\
\hline Days of hospitalization before bacteremia & $18(5-34)$ & $21(4.5-48)$ & $16(5.8-32)$ & 0.161 \\
\hline \multicolumn{5}{|l|}{ Comorbidity } \\
\hline Alcoholism & $30(7.9)$ & $9(10.1)$ & $21(7.2)$ & 0.514 \\
\hline Liver cirrhosis & $23(6.1)$ & $5(5.6)$ & $18(6.2)$ & 1.000 \\
\hline Chronic kidney disease & $90(23.7)$ & $28(31.5)$ & $62(21.4)$ & 0.070 \\
\hline Type 2 diabetes mellitus & $99(26.1)$ & $20(22.5)$ & $79(27.2)$ & 0.448 \\
\hline Chronic obstructive pulmonary disease & $68(17.9)$ & $17(19.1)$ & $51(17.6)$ & 0.867 \\
\hline Hypertension & $127(33.5)$ & $31(34.8)$ & $96(33.1)$ & 0.862 \\
\hline Coronary artery disease & $56(14.8)$ & $22(24.7)$ & $34(11.7)$ & 0.004 \\
\hline Congestive heart failure & $43(11.3)$ & $13(14.6)$ & $30(10.3)$ & 0.359 \\
\hline Cerebral vascular accident & $71(18.7)$ & $17(19.1)$ & $58(20.0)$ & 0.973 \\
\hline Collagen vascular disease & $18(4.7)$ & $5(5.6)$ & $13(4.5)$ & 0.775 \\
\hline Malignancy & $128(33.8)$ & $28(31.5)$ & $100(34.5)$ & 0.690 \\
\hline Neutropenia & $18(4.7)$ & $4(4.5)$ & $14(4.8)$ & 1.000 \\
\hline Chemotherapy & $39(10.3)$ & $6(6.7)$ & $33(11.4)$ & 0.289 \\
\hline Immunosuppressive therapy & $19(5.0)$ & $4(4.5)$ & $15(5.2)$ & 1.000 \\
\hline Recent surgery (within 4 weeks) & $126(33.2)$ & $26(29.2)$ & $100(34.5)$ & 0.427 \\
\hline Trauma & $11(2.9)$ & $1(1.1)$ & $10(3.4)$ & 0.470 \\
\hline Shock within 3 days & $15(4.0)$ & $0(0.0)$ & $15(5.2)$ & 0.027 \\
\hline APACHE II score within $24 \mathrm{~h}$ before bacteremia onset & $24(18-31)$ & $24(18.3-31)$ & $24(27-31)$ & 0.820 \\
\hline \multicolumn{5}{|l|}{ Invasive procedures ${ }^{a}$} \\
\hline Abdominal drainage & $33(8.7)$ & $3(3.4)$ & $30(10.3)$ & 0.068 \\
\hline Arterial catheter & $76(20.1)$ & $15(16.9)$ & $61(21.0)$ & 0.478 \\
\hline Central venous catheter & $196(51.7)$ & $51(57.3)$ & $145(50.2)$ & 0.291 \\
\hline Foley catheter & $221(58.3)$ & $53(59.6)$ & $168(57.9)$ & 0.882 \\
\hline Hemodialysis & $27(7.1)$ & $8(9.0)$ & $19(6.6)$ & 0.585 \\
\hline Nasogastric tube & $255(67.3)$ & $63(70.8)$ & $192(66.2)$ & 0.499 \\
\hline Pulmonary artery catheter & $41(10.8)$ & $7(7.9)$ & $34(11.7)$ & 0.406 \\
\hline Total parental nutrition & $27(7.1)$ & $2(2.2)$ & $25(8.6)$ & 0.070 \\
\hline Tracheotomy & $46(12.1)$ & $16(18.0)$ & $30(10.3)$ & 0.081 \\
\hline Ventilator & $192(50.7)$ & $51(57.3)$ & $141(48.6)$ & 0.190 \\
\hline \multicolumn{5}{|l|}{ Infection source ${ }^{b}$} \\
\hline Respiratory tract & $213(56.2)$ & $54(60.7)$ & $159(54.8)$ & 0.395 \\
\hline Urinary tract & $31(8.2)$ & $4(4.5)$ & $27(9.3)$ & 0.219 \\
\hline Catheter-related & $35(9.2)$ & $11(12.4)$ & $24(8.3)$ & 0.340 \\
\hline Intra-abdomen & $26(6.9)$ & $3(3.4)$ & $23(7.9)$ & 0.212 \\
\hline Soft tissue or wound & $12(3.2)$ & $4(4.5)$ & $8(2.8)$ & 0.487 \\
\hline Primary bacteremia & $71(18.7)$ & $16(18.0)$ & $55(19.0)$ & 0.957 \\
\hline \multicolumn{5}{|l|}{ Resistance profiles of the bloodstream isolate } \\
\hline Multidrug resistance & $265(69.9)$ & $66(74.2)$ & $199(68.6)$ & 0.387 \\
\hline Carbapenem resistance & $91(24.0)$ & $19(21.3)$ & $72(24.8)$ & 0.596 \\
\hline Appropriate antimicrobial therapy & $124(32.7)$ & $12(13.5)$ & $112(38.6)$ & $<0.001$ \\
\hline 14-day mortality & $104(27.4)$ & $26(29.2)$ & $78(26.9)$ & 0.770 \\
\hline 30-day mortality & $135(35.6)$ & $34(38.2)$ & $101(34.8)$ & 0.561 \\
\hline
\end{tabular}

NOTE. Data are median values (interquartile range) for continuous variables and numbers of cases (\%) for categorical variables. ICU, intensive care unit; APACHE II, Acute Physiologic and Chronic Health Evaluation II. ${ }^{\text {a }}$ At the time that blood culture was obtained. ${ }^{b}$ Patients may have more than one source of bacteremia.

Logistic regression analysis was performed to determine the risk factors for 14-day mortality in the entire population with $A$. baumannii bacteremia, as shown in Table 2. Polymicrobial bacteremia was not independently associated with increased 14-day mortality. Malignancy, shock, and higher APACHE II score were independent predictors of 14-day mortality, whereas appropriate antimicrobial therapy was independently associated with reduced 14-day mortality $(p=0.001)$. The predictors for 
30-day mortality among patients with A. baumannii bacteremia were analyzed in the same fashion as the above analyses. The results were similar to those for prediction of 14-day mortality (Table S1).

Table 2. Logistic regression analysis of predictors for 14-day mortality among patients with Acinetobacter baumannii bacteremia.

\begin{tabular}{ccccc}
\hline Demographic or Characteristic & \multicolumn{2}{c}{ Univariable Analysis } & \multicolumn{2}{c}{ Multivariable Analysis } \\
\hline & Odds Ratio (95\% CI) & $p$ & Odds Ratio (95\% CI) & $p$ \\
\hline Chronic kidney disease & $1.617(0.946-2.765)$ & 0.079 & & \\
Coronary artery disease & $1.735(0.956-3.148)$ & 0.070 & & \\
Malignancy & $1.667(1.046-2.657)$ & 0.032 & $2.554(1.373-4.753)$ & 0.003 \\
Neutropenia & $3.551(1.361-9.263)$ & 0.010 & & \\
Recent surgery (within 4 weeks) & $0.410(0.239-0.701)$ & 0.001 & & \\
Shock within 3 days & $4.081(2.386-6.981)$ & $<0.001$ & $2.430(1.220-4.843)$ & 0.012 \\
APACHE II score & $1.169(1.129-1.210)$ & $<0.001$ & $1.173(1.130-1.216)$ & $<0.001$ \\
Arterial catheter & $2.170(1.280-3.680)$ & 0.004 & & \\
Central venous catheter & $1.682(1.060-2.668)$ & 0.027 & & \\
Foley catheter & $2.285(1.400-3.729)$ & 0.001 & & \\
Nasogastric tube & $2.198(1.293-3.735)$ & 0.004 & & \\
Ventilator & $2.054(1.291-3.267)$ & 0.002 & & \\
Respiratory tract infection & $2.015(1.252-3.243)$ & 0.004 & & \\
Urinary tract infection & $0.262(0.078-0.881)$ & 0.030 & & \\
Appropriate antimicrobial therapy & $0.525(0.313-0.881)$ & 0.015 & $0.317(0.162-0.621)$ & \\
Polymicrobial bacteremia & $1.122(0.663-1.897)$ & 0.668 & & \\
\hline
\end{tabular}

Abbreviations: ICU, intensive care unit; APACHE II, Acute Physiologic and Chronic Health Evaluation II; CI, confidence interval.

We stratified the 89 patients with polymicrobial A. baumannii bacteremia according to the concomitantly isolated bacterial species (Table 3). Coagulase-negative staphylococci were the most common isolated microorganisms (20 cases). Among them, 17 patients $(85.0 \%)$ were caused by a device-related infection. Nine patients $(52.9 \%)$ had their infected device removed at a median duration of six days (interquartile range, 4-8) after the onset of the bacteremia. However, the removal of device did not affect their clinical outcome. There were 12 patients with polymicrobial A. baumannii bacteremia that received appropriate antimicrobial therapy. Half of them received combination therapy. Carbapenem plus vancomycin/teicoplanin was the most frequently used antimicrobial regimen for those with polymicrobial A. baumannii bacteremia (Table S2).

Although no significant difference in 14-day mortality rates was observed between patients with polymicrobial and monomicrobial A. baumannii bacteremia, the rate in polymicrobial A. baumannii bacteremia varied according to the concomitant pathogen. Concomitant Escherichia coli bacteremia had the highest 14 -day mortality rate $(71.4 \%)$, followed by Pseudomonas aeruginosa $(50.0 \%)$ and Enterobacter spp. bacteremia (37.5\%). The 14-day mortality rate was higher in patients who had polymicrobial $A$. baumannii bacteremia with concomitant Gram-negative bacilli (GNB) than in patients with concomitant Gram-positive cocci (GPC) (Table 3; 38.9\% vs. $19.1 \%, p=0.053$ ) (Figure 1; $p=0.038$, log-rank test). The rates of methicillin-resistant Staphylococcus aureus (MRSA), extended-spectrum beta-lactamase (ESBL)-producing K. pneumoniae, ESBL-producing E. coli, and AmpC-producing E. coli were $80.0 \%, 16.7 \%, 14.3 \%$, and $14.3 \%$, respectively (Table S3). The presence of these resistant bacteria was not associated with significantly higher 14-day mortality. 
Table 3. Characteristics of patients with polymicrobial Acinetobacter baumannii bacteremia stratified by concomitantly isolated bacterial species.

\begin{tabular}{|c|c|c|c|c|c|}
\hline \multirow[b]{2}{*}{$\begin{array}{l}\text { Concomitantly Isolated } \\
\text { Bacterial Species }\end{array}$} & \multirow[b]{2}{*}{$\begin{array}{l}\text { No. }(\%) \text { of } \\
\text { Patients }\end{array}$} & \multirow{2}{*}{$\begin{array}{c}\text { APACHE II Score, } \\
\text { Mean (Interquartile } \\
\text { Range) }\end{array}$} & \multicolumn{3}{|c|}{ No. (\%) of Patients } \\
\hline & & & $\begin{array}{c}\text { Appropriate } \\
\text { Antimicrobial } \\
\text { Therapy }\end{array}$ & $\begin{array}{l}\text { Concomitant } \\
\text { MDR/CR } A \text {. } \\
\text { baumannii }\end{array}$ & $\begin{array}{c}\text { 14-Day } \\
\text { Non-Survival }\end{array}$ \\
\hline Gram-positive cocci (GPC) & $47(52.8)$ & $23(18-28)$ & $5(10.6)$ & $39 / 8(83.0 / 17.0)$ & $9(19.1)$ \\
\hline $\begin{array}{l}\text { Coagulase-negative } \\
\text { staphylococci (CNS) }\end{array}$ & $20(22.5)$ & $18.5(11.25-25.75)$ & $1(5.0)$ & $15 / 3(78.0 / 15.0)$ & $2(10.0)$ \\
\hline Enterococcus spp. & $12(13.5)$ & $24.5(18.5-37.75)$ & $2(16.7)$ & $10 / 0(83.3 / 0)$ & $3(25.0)$ \\
\hline Staphylococcus aureus & $4(4.5)$ & $24.5(22-35.25)$ & $1(25.0)$ & $4 / 2(100.0 / 50.0)$ & $1(25.0)$ \\
\hline $\begin{array}{c}\text { Enterococcus spp. }+S . \\
\text { aureus }\end{array}$ & $4(4.5)$ & $26.5(9.25-28.75)$ & $0(0)$ & $3 / 1(75.0 / 25.0)$ & $0(0)$ \\
\hline $\mathrm{CNS}+\mathrm{S}$. aureus & $2(2.3)$ & 19,24 & $0(0)$ & $1 / 1(50.0 / 50.0)$ & $0(0)$ \\
\hline Other GPC ${ }^{\mathrm{a}}$ & $5(5.6)$ & $25(22.5-30.5)$ & $1(20.0)$ & $5 / 1(100.0 / 20.0)$ & $3(60.0)$ \\
\hline $\begin{array}{l}\text { Gram-negative bacilli } \\
\text { (GNB) }\end{array}$ & $36(40.5)$ & $24(19-35)$ & $6(16.7)$ & $23 / 10(63.9 / 27.8)$ & $14(38.9)$ \\
\hline Pseudomonas aeruginosa & $8(9.0)$ & $33.5(18.25-38.75)$ & $2(25.0)$ & $6 / 2(75.0 / 25.0)$ & $4(50.0)$ \\
\hline Enterobacter spp. & $8(9.0)$ & $26(22.25-40)$ & $1(12.5)$ & $5 / 2(62.5 / 25.0)$ & $3(37.5)$ \\
\hline Escherichia coli & $7(7.9)$ & $31(24-39)$ & $1(14.3)$ & $6 / 2(85.7 / 28.6)$ & $5(71.4)$ \\
\hline Klebsiella pneumoniae & $6(6.7)$ & $20(17.5-31.5)$ & $0(0)$ & $3 / 1(50.0 / 16.7)$ & $1(16.7)$ \\
\hline Other GNB ${ }^{\mathrm{b}}$ & $7(7.9)$ & $18(10-27)$ & $2(28.6)$ & $4 / 4(42.9 / 42.9)$ & $1(14.3)$ \\
\hline Yeast $^{\mathrm{c}}$ & $3(3.4)$ & $18,23,33$ & $1(33.3)$ & $2 / 1(66.7 / 33.3)$ & $1(33.3)$ \\
\hline Mixed GNB and GPC & $3(3.4)$ & $19,24,47$ & $0(0)$ & $2 / 0(66.7 / 0)$ & $2(66.7)$ \\
\hline
\end{tabular}

Abbreviations: APACHE II, Acute Physiologic and Chronic Health Evaluation II; MDR, multidrug resistance; CR, carbapenem resistance. a Includes Aerococcus $(n=1)$, concomitant Coagulase-negative staphylococci and Enterococcus spp. $(n=1)$, Lactobacillus spp. $(n=1)$, Listeria $(n=1)$, and Streptococcus spp. $(n=1) .{ }^{\mathrm{b}}$ Includes Aeromonas sobria $(n=1)$, Alcaligenes xylosoxidans $(n=1)$, Chryseobacterium indologens $(n=1)$, Citrobacter diversus $(n=1)$, Pantoea agglomerans $(n=1)$, Proteus mirabilis $(n=1)$, and Serratia marcescens $(n=1) .{ }^{c}$ Includes Candida glabrata $(n=1)$; Candida tropicalis $(n=1)$; and concomitant coagulase-negative staphylococci, Staphylococcus aureus, and Candida albicans $(n=1)$.

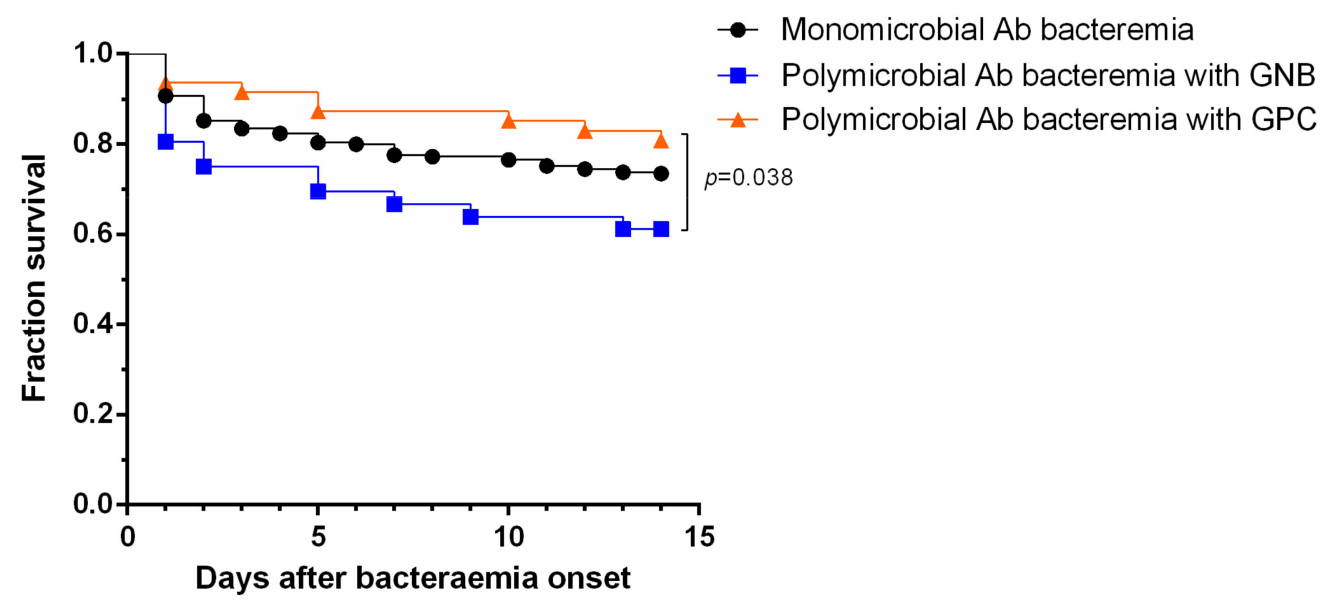

Figure 1. Kaplan-Meier plot showing the survival rates of patients with acquired monomicrobial Acinetobacter baumannii (Ab) bacteremia, polymicrobial Ab bacteremia with concomitant Gram-negative bacilli (GNB), and polymicrobial Ab bacteremia with concomitant Gram-positive cocci (GPC) (polymicrobial Ab bacteremia with concomitant GNB versus monomicrobial Ab bacteremia, $p=0.098$ by log-rank test; polymicrobial Ab bacteremia with concomitant GPC versus monomicrobial $\mathrm{Ab}$ bacteremia, $p=0.265$ by log-rank test).

To investigate the influence of $A$. baumannii antibiotic resistance on the mortality of patients with polymicrobial bacteremia with concomitant GNB infection, we extracted data of those with multidrug-resistant $A$. baumannii (MDRAb) or carbapenem-resistant $A$. baumannii (CRAb) infections for further analysis. Patients with MDRAb and concomitant GNB bacteremia had a higher 14-day mortality rate than patients with monomicrobial MDRAb bacteremia (Figure $2 ; p=0.036$, log-rank test). Patients with CRAb and concomitant GNB bacteremia had a higher 14-day mortality rate than 
patients with monomicrobial CRAb bacteremia $(50.0 \%$ vs. $30.6 \%, p=0.285)$. The difference was not statistically significant in this small cohort $(n=82)$.

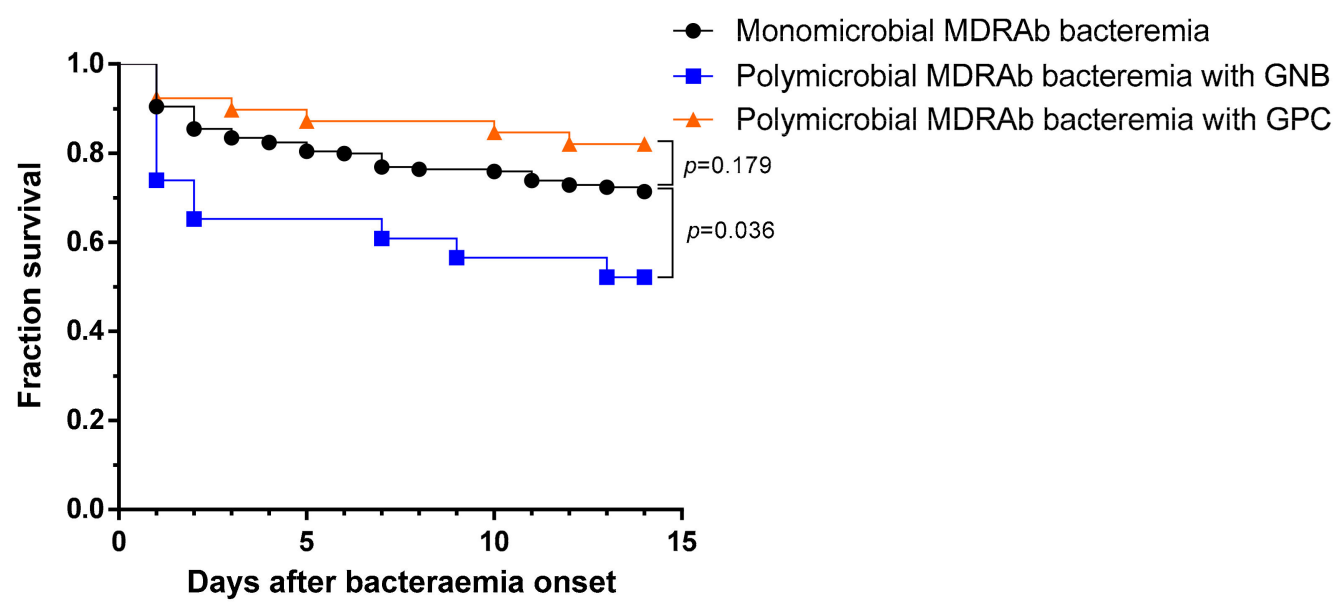

Figure 2. Kaplan-Meier plot showing the survival rates of patients with acquired polymicrobial multidrug-resistant Acinetobacter baumannii (MDRAb) bacteremia with concomitant Gram-negative bacilli (GNB), polymicrobial Ab bacteremia with concomitant Gram-positive cocci (GPC), and those with acquired monomicrobial MDRAb bacteremia. $p$-values were calculated by log-rank tests. (polymicrobial MDRAb bacteremia with concomitant GNB versus polymicrobial MDRAb bacteremia with concomitant GPC, $p=0.009$ by log-rank test).

The survival analysis at 30 days was analyzed in the same fashion as the above analyses (Figures S1 and S2). The results were similar to those for 14-day mortality.

\section{Discussion}

While polymicrobial A. baumannii bacteremia is not uncommon, its clinical significance has not previously been elucidated. This retrospective study assessed the differences between monomicrobial and polymicrobial A. baumannii bacteremia and identified possible independent risk factors for 14-day mortality for these infections. We found that polymicrobial bacteremia was not an independent predictor of mortality, whereas appropriate antimicrobial therapy was independently associated with reduced mortality.

This study, in agreement with previous studies $[6,9,11,12]$, observed that approximately one-fourth of patients with A. baumannii bloodstream infections presented with polymicrobial bacteremia. Owing to its high prevalence, investigation of the clinical significance of polymicrobial A. baumannii bacteremia is warranted. These patients were older and more likely to have coronary artery disease and experienced shock at the time of bacteremia. Moreover, as few as one out of seven patients received appropriate antimicrobial therapy directed against every microbial species isolated from the blood. As the clinical characteristics differed between monomicrobial and polymicrobial A. baumannii bacteremia, studies on the clinical outcomes of patients with monomicrobial and polymicrobial A. baumannii bacteremia should evaluate the infections separately. Furthermore, when evaluating the efficacy of various regimens on the clinical outcome of A. baumannii bacteremia, polymicrobial A. baumannii bacteremia should be excluded, unless the antibiotic efficacy against the concomitant isolated microorganisms was also evaluated.

Our results showed that the 14-day mortality rate was not significantly higher in patients with polymicrobial than in patients with monomicrobial A. baumannii bacteremia. This finding is consistent with those of previous studies and suggests that polymicrobial infection was not an independent risk factor for mortality of $A$. baumannii bacteremia [10,11,22,23]. Although several studies have reported higher mortality rates in patients with polymicrobial bacteremia $[3,5,6,24]$, the attributable mortality rate of polymicrobial bacteremia varied among causative pathogens $[3,25,26]$. Compared with 
monomicrobial bacteremia, polymicrobial bacteremia involving Staphylococcus aureus [27], Enterococcus spp. [28], and P. aeruginosa [29] have higher mortality rates. In contrast, polymicrobial Klebsiella pneumoniae bacteremia did not result in a worse prognosis [30]. These findings indicate that the influence of polymicrobial bacteremia on clinical prognosis should be investigated separately according to the causative pathogens.

The interbacterial interactions in polymicrobial infection, which vary among co-existing species, include metabolite exchange, co-aggregation, interspecies signaling, antibiotic resistance, and biofilm formation [31]. While most co-existing bacteria promote their pathogenesis through these interactions, some of them exhibit a competitive relationship [31]. Thus, these interbacterial interactions in polymicrobial infection may have two-sided effects on the disease severity. Therefore, the different co-existing bacteria in polymicrobial infections may have diverse effects on the clinical outcome. This in line with our findings that different concomitant bacterial pathogens had heterogeneous clinical outcomes. Compared with monomicrobial A. baumannii bacteremia, the higher mortality rate observed in patients with concomitant GNB bacteremia and lower mortality rate in those with concomitant GPC bacteremia suggested that the interactions between A. baumannii and GNB had negative effects, while those between $A$. baumannii and GPC positively influenced the disease severity.

On the basis of previous studies showing that beta-lactamase-producing pathogens can provide indirect pathogenesis by protecting the other pathogens in polymicrobial infection environments [13], we assessed whether the co-existence of drug-resistant $A$. baumannii would protect the concomitant GNB and result in a worse clinical outcome. Our findings were consistent with those of previous studies reporting higher 14-day mortality in patients with polymicrobial bacteremia with concomitant MDRAb and GNB than in those with monomicrobial MDRAb bacteremia. Although patients with polymicrobial bacteremia with concomitant CRAb and GNB had a higher 14-day mortality rate than those with monomicrobial CRAb bacteremia, the difference was not statistically significant in this small population.

Previous studies of monomicrobial A. baumannii bacteremia also reported inappropriate antimicrobial therapy to be an independent risk factor for mortality $[9,11,12,22,32]$. The results of the present study strengthened these findings by enrolling a large number of patients and including a number of cases with polymicrobial A. baumannii bacteremia. Ours and previous studies consistently found that inappropriate antimicrobial therapy was significantly associated with survival in patients with acquired polymicrobial bacteremia [2]. When studies evaluating the efficacy of appropriate antimicrobial therapy enroll both monomicrobial and polymicrobial A. baumannii bacteremia, appropriate therapy should be defined as a single or a combination of antimicrobial agents covering all isolated microbes.

The weakness of this study was the retrospective design required to enroll sufficient patients for detailed analysis at a single tertiary care medical center. The major strength of our study was its inclusion of a number of patients with genomically-defined A. baumannii bacteremia, consistency of patient care in a single center, stringent definition of the appropriateness of antimicrobial therapy, and a well-defined primary endpoint of 14-day mortality. We also conducted multivariate analysis to assess clinical variables that were significantly associated with mortality owing to A. baumannii bacteremia.

\section{Conclusions}

Polymicrobial A. baumannii bacteremia was not an independent risk factor for 14-day mortality. Regardless of monomicrobial or polymicrobial infection, appropriate antimicrobial therapy remains an important life-saving measure for $A$. baumannii bacteremia patients.

Supplementary Materials: The following are available online at http://www.mdpi.com/2077-0383/9/1/153/s1, Table S1: Logistic regression analysis of predictors for 30-day mortality among patients with Acinetobacter baumannii bacteremia. Table S2: Concomitantly isolated bacterial species and the appropriate antimicrobial regimens used for polymicrobial Acinetobacter baumannii bacteremia. Table S3: Resistant rates of the concomitant pathogens in polymicrobial bacteremia. Figure S1: Kaplan-Meier plot showing the survival rates at 30 days of patients with acquired monomicrobial Acinetobacter baumannii $(\mathrm{Ab})$ bacteremia, polymicrobial Ab bacteremia 
with concomitant Gram-negative bacilli and polymicrobial Ab bacteremia with concomitant Gram-positive cocci. Figure S2: Kaplan-Meier plot showing the survival rates of patients with acquired polymicrobial multidrug-resistant Acinetobacter baumannii (MDRAb) bacteremia with concomitant Gram-negative bacilli (GNB), polymicrobial $\mathrm{Ab}$ bacteremia with concomitant Gram-positive cocci (GPC), and those with acquired monomicrobial MDRAb bacteremia.

Author Contributions: Conceptualization: Y.-C.W., T.-L.C., F.-D.W., and Y.-T.L. Data curation: Y.-C.W., W.-W.K., and Y.-S.Y. Formal analysis: W.-W.K., C.C.-K., and F.-Y.K. Funding acquisition: Y.-C.W., Y.-S.Y., and Y.-T.L. Investigation: Y.-C.W., T.-L.C., and Y.-T.L. Methodology: Y.-S.Y., S.-C.K., C.-H.C., and T.-L.C. Project administration: T.-L.C., F.-D.W., and Y.-T.L. Resources: W.-W.K., S.-C.K., and C.-H.C. Software: Y.-S.Y., C.C.-K., and F.-Y.K. Supervision: T.-L.C., F.-D.W., and Y.-T.L., Validation: Y.-T.L. Visualization: W.-W.K. and Y.-S.Y. Writing-original draft: Y.-C.W., W.-W.K., and Y.-T.L. Writing-review \& editing: Y.-C.W., T.-L.C., and Y.-T.L. All authors have read and agreed to the published version of the manuscript.

Funding: This work was supported by grants from the Taipei Veterans General Hospital (grant numbers V106B-002, V107C-037, V108C-012, VTA106-T-5-3, VTA107-T-3-2, and VTA108-T-2-3), Tri-Service General Hospital (grant numbers TSGH-C107-099, TSGH-E-109237, and VTA108-T-2-2), Ministry of National Defense-Medical Affairs Bureau (grant numbers MAB-107-095 and MAB-108-038), and Ministry of Science and Technology (grant numbers MOST 105-2314-B-016-039-MY3, MOST 107-2314-B-075-066-MY3, MOST 107-2314-B-016-051-MY3, and MOST 108-2314-B-016-029). The funders had no role in the study design, data collection and analysis, decision to publish, or preparation of the manuscript.

Conflicts of Interest: The authors declare no conflicts of interest.

\section{References}

1. Hermans, P.E.; Washington, J.A., 2nd. Polymicrobial bacteremia. Ann. Intern. Med. 1970, 73, $387-392$. [CrossRef] [PubMed]

2. Roselle, G.A.; Watanakunakorn, C. Polymicrobial bacteremia. JAMA 1979, 242, 2411-2413. [CrossRef] [PubMed]

3. Weinstein, M.P.; Murphy, J.R.; Reller, L.B.; Lichtenstein, K.A. The clinical significance of positive blood cultures: A comprehensive analysis of 500 episodes of bacteremia and fungemia in adults. II. Clinical observations, with special reference to factors influencing prognosis. Rev. Infect. Dis. 1983, 5, 54-70. [CrossRef] [PubMed]

4. Cooper, G.S.; Havlir, D.S.; Shlaes, D.M.; Salata, R.A. Polymicrobial bacteremia in the late 1980s: Predictors of outcome and review of the literature. Medicine 1990, 69, 114-123. [CrossRef]

5. McKenzie, F.E. Case mortality in polymicrobial bloodstream infections. J. Clin. Epidemiol. 2006, 59, 760-761. [CrossRef]

6. Pavlaki, M.; Poulakou, G.; Drimousis, P.; Adamis, G.; Apostolidou, E.; Gatselis, N.K.; Kritselis, I.; Mega, A.; Mylona, V.; Papatsoris, A.; et al. Polymicrobial bloodstream infections: Epidemiology and impact on mortality. J Glob Antimicrob Resist 2013, 1, 207-212. [CrossRef]

7. Reuben, A.G.; Musher, D.M.; Hamill, R.J.; Broucke, I. Polymicrobial bacteremia: Clinical and microbiologic patterns. Rev. Infect. Dis. 1989, 11, 161-183. [CrossRef]

8. Peleg, A.Y.; Seifert, H.; Paterson, D.L. Acinetobacter baumannii: Emergence of a successful pathogen. Clin. Microbiol. Rev. 2008, 21, 538-582. [CrossRef]

9. Lee, Y.T.; Kuo, S.C.; Yang, S.P.; Lin, Y.T.; Tseng, F.C.; Chen, T.L.; Fung, C.P. Impact of appropriate antimicrobial therapy on mortality associated with Acinetobacter baumannii bacteremia: Relation to severity of infection. Clin. Infect. Dis. 2012, 55, 209-215. [CrossRef]

10. Du, X.; Xu, X.; Yao, J.; Deng, K.; Chen, S.; Shen, Z.; Yang, L.; Feng, G. Predictors of mortality in patients infected with carbapenem-resistant Acinetobacter baumannii: A systematic review and meta-analysis. Am J. Infect. Control 2019, 47, 1140-1145. [CrossRef]

11. Cisneros, J.M.; Reyes, M.J.; Pachon, J.; Becerril, B.; Caballero, F.J.; Garcia-Garmendia, J.L.; Ortiz, C.; Cobacho, A.R. Bacteremia due to Acinetobacter baumannii: Epidemiology, clinical findings, and prognostic features. Clin. Infect. Dis. 1996, 22, 1026-1032. [CrossRef] [PubMed]

12. Cisneros, J.M.; Rodriguez-Bano, J. Nosocomial bacteremia due to Acinetobacter baumannii: Epidemiology, clinical features and treatment. Clin. Microbiol. Infect. 2002, 8, 687-693. [CrossRef] [PubMed]

13. Liao, Y.T.; Kuo, S.C.; Lee, Y.T.; Chen, C.P.; Lin, S.W.; Shen, L.J.; Fung, C.P.; Cho, W.L.; Chen, T.L. Sheltering effect and indirect pathogenesis of carbapenem-resistant Acinetobacter baumannii in polymicrobial infection. Antimicrob. Agents Chemother. 2014, 58, 3983-3990. [CrossRef] [PubMed]

14. CLSI. Principles and procedures for blood cultures: Approved guideline; M47-A; Clinical and Laboratory Standards Institute: Wayne, PA, USA, 2007. 
15. Chen, H.P.; Chen, T.L.; Lai, C.H.; Fung, C.P.; Wong, W.W.; Yu, K.W.; Liu, C.Y. Predictors of mortality in Acinetobacter baumannii bacteremia. J. Microbiol. Immunol. Infect. 2005, 38, 127-136. [PubMed]

16. Kuo, S.C.; Lee, Y.T.; Yang, S.P.; Chiang, M.C.; Lin, Y.T.; Tseng, F.C.; Chen, T.L.; Fung, C.P. Evaluation of the effect of appropriate antimicrobial therapy on mortality associated with Acinetobacter nosocomialis bacteraemia. Clin. Microbiol. Infect. 2013, 19, 634-639. [CrossRef]

17. Knaus, W.A.; Draper, E.A.; Wagner, D.P.; Zimmermanm, J.E. APACHE II: A severity of disease classification system. Crit. Care Med. 1985, 13, 818-829. [CrossRef]

18. Garner, J.S.; Jarvis, W.R.; Emori, T.G.; Horan, T.C.; Hughes, J.M. CDC definitions for nosocomial infections, 1988. Am. J. Infect. Control 1988, 16, 128-140. [CrossRef]

19. Chen, T.L.; Siu, L.K.; Wu, R.C.; Shaio, M.F.; Huang, L.Y.; Fung, C.P.; Lee, C.M.; Cho, W.L. Comparison of one-tube multiplex PCR, automated ribotyping and intergenic spacer (ITS) sequencing for rapid identification of Acinetobacter baumannii. Clin. Microbiol. Infect. 2007, 13, 801-806. [CrossRef]

20. Methods for dilution antimicrobial susceptibility tests for bacteria that grow aerobically; approved standard-9th ed. M07-A9; Clinical and Laboratory Standards Institute: Wayne, PA, USA, 2012.

21. Performance standards for antimicrobial susceptibility testing: 27th informational supplement M100-S27; Clinical and Laboratory Standards Institute: Wayne, PA, USA, 2017.

22. Esterly, J.S.; Griffith, M.; Qi, C.; Malczynski, M.; Postelnick, M.J.; Scheetz, M.H. Impact of carbapenem resistance and receipt of active antimicrobial therapy on clinical outcomes of Acinetobacter baumannii bloodstream infections. Antimicrob. Agents Chemother. 2011, 55, 4844-4849. [CrossRef]

23. Cheng, A.; Chuang, Y.C.; Sun, H.Y.; Sheng, W.H.; Yang, C.J.; Liao, C.H.; Hsueh, P.R.; Yang, J.L.; Shen, N.J.; Wang, J.T.; et al. Excess mortality associated with colistin-tigecycline compared with colistin-carbapenem combination therapy for extensively drug-resistant Acinetobacter baumannii bacteremia: A multicenter prospective observational study. Crit. Care Med. 2015, 43, 1194-1204. [CrossRef]

24. Weinstein, M.P.; Towns, M.L.; Quartey, S.M.; Mirrett, S.; Reimer, L.G.; Parmigiani, G.; Reller, L.B. The clinical significance of positive blood cultures in the 1990s: A prospective comprehensive evaluation of the microbiology, epidemiology, and outcome of bacteremia and fungemia in adults. Clin. Infect. Dis. 1997, 24, 584-602. [CrossRef] [PubMed]

25. Ilavska, I.; Pichna, P.; Stopkova, K.; Grausova, S.; Krupova, I.; Oravcova, E.; Koren, P.; Lacka, J.; Studena, M.; Spanik, S.; et al. Polymicrobial bacteremia in cancer patients: Analysis of risk factors, etiology and outcome in 214 episodes. Int. J. Antimicrob. Agents 1996, 7, 101-107. [CrossRef]

26. Roberts, F.J.; Geere, I.W.; Coldman, A. A three-year study of positive blood cultures, with emphasis on prognosis. Rev. Infect. Dis. 1991, 13, 34-46. [CrossRef] [PubMed]

27. Park, S.Y.; Park, K.H.; Bang, K.M.; Chong, Y.P.; Kim, S.H.; Lee, S.O.; Choi, S.H.; Jeong, J.Y.; Woo, J.H.; Kim, Y.S.; et al. Clinical significance and outcome of polymicrobial Staphylococcus aureus bacteremia. J. Infect. 2012, 65, 119-127. [CrossRef] [PubMed]

28. Falcone, M.; Tiseo, G.; Dentali, F.; Foglia, E.; Campanini, M.; Menichetti, F.; Mazzone, A. Early alert from the microbiology laboratory improves the outcome of elderly patients with Enterococcus spp. bloodstream infection: Results from a multicenter prospective study. J. Glob. Antimicrob. Resist. 2019, 18, 139-144. [CrossRef] [PubMed]

29. Aliaga, L.; Mediavilla, J.D.; Llosa, J.; Miranda, C.; Rosa-Fraile, M. Clinical significance of polymicrobial versus monomicrobial bacteremia involving Pseudomonas aeruginosa. Eur. J. Clin. Microbiol. Infect. Dis. 2000, 19, 871-874. [CrossRef]

30. Liu, Q.; Wu, J.; Wang, Z.; Wu, X.; Wang, G.; Ren, J. Polymicrobial bacteremia involving Klebsiella pneumoniae in patients with complicated intra-abdominal infections: Frequency, co-pathogens, risk factors, and clinical outcomes. Surg. Infect. (Larchmt) 2019, 20, 317-325. [CrossRef]

31. Peters, B.M.; Jabra-Rizk, M.A.; O'May, G.A.; Costerton, J.W.; Shirtliff, M.E. Polymicrobial interactions: Impact on pathogenesis and human disease. Clin. Microbiol. Rev. 2012, 25, 193-213. [CrossRef]

32. Wang, Y.C.; Huang, T.W.; Yang, Y.S.; Kuo, S.C.; Chen, C.T.; Liu, C.P.; Liu, Y.M.; Chen, T.L.; Chang, F.Y.; $\mathrm{Wu}, \mathrm{S} . \mathrm{H} . ;$ et al. Biofilm formation is not associated with worse outcome in Acinetobacter baumannii bacteremic pneumonia. Sci. Rep. 2018, 8, 7289. [CrossRef]

(C) 2020 by the authors. Licensee MDPI, Basel, Switzerland. This article is an open access article distributed under the terms and conditions of the Creative Commons Attribution (CC BY) license (http://creativecommons.org/licenses/by/4.0/). 